\title{
Heterogeneity and New Epitopes of Hepatitis C Virus Genotype 4
}

\author{
Moataza H. Omran ${ }^{1, *}$, Wael Nabil ${ }^{1}$, Samar S. Youssef ${ }^{1}$, Mervat El-Sayed ${ }^{2}$, Mostafa K. El Awady ${ }^{1}$ \\ ${ }_{2}^{1}$ Microbial Biotechnology Department, Genetic Engineering Division, National Research Centre, Dokki Cairo, Egypt \\ ${ }^{2}$ Chemistry Department, Faculty of Science, Cairo University, Dokki Cairo, Egypt \\ ${ }^{*}$ Corresponding author: Moataza H. Omran, Microbial Biotechnology Department, Genetic Engineering Division, National Research Centre, Dokki Cairo, Egypt. Tel: +20-1223735450, \\ Fax:+20-233370931, E-mail: moataza13@yahoo.com, amohammadee@gmail.com.
}

Received: January 31, 2013; Revised: April 14, 2013; Accepted: Jun 20, 2013

\begin{abstract}
Background: Hepatitis C virus (HCV) was found to have a major role in human liver disease by its ability to face the host-cell defenses and the immune system. Heterogeneity of HCV was the key for its adaptation to its host and represented a significant hurdle for the development of both effective vaccines as well as for novel therapeutic interventions.

Objectives: Due to the heterogeneity of HCV virus because of both high replication and high mutation rate in vivo, this study was conducted to analyze different isolates of Egyptian patients of genotype 4, of the most mutant regions of the virus (E1 and E2) as they played an important role in viral persistence by escaping from the immune system of the host body.

Patients and Methods: This study was conducted through PCRamplification of E1 and E2 regions, sequencing and phylogenetic analysis, calculating synonyms and non-synonyms substitutions, finding the possible glycosylation sites and different epitope domains.

Results: The present work figured out that the heterogeneity of the quasispecies of our local strains 4 a was high showing up $15 \%$ diversity. This study also showed four glycosylation sites that play an important role in the entry of the virus and protein folding. Besides, different epitpoes were identified in different regions of the E1 and E2 domains; a finding which would help in determining the neutralizing and non-neutralizing antibodies.

Conclusions: This study would help in understanding the driving forces of genetic diversity and would be fundamental for representing potential candidate targets for antibodies and the development of vaccine trials.
\end{abstract}

Keywords: Hepacivirus; Phylogenetic Analysis; Polysaccharides; Epitopes

\section{Background}

Hepatitis C virus (HCV) infection is a serious health problem that affects people worldwide. It was figured outthat around 25\% of Egyptians are infected by the virus and only $20 \%$ of the acutely infected patients might have spontaneous viral clearance. Most of the patients who had chronic hepatitis might then progress to liver cirrhosis and hepatocellular carcinoma. Current therapies of interferon + ribavirin for HCV of genotype 4 were not successful and did not exceed more than 50\% and thus finding a cure for this disease is of great importance (1, 2). Hepatitis $\mathrm{C}$ virus (HCV) is a positive-stranded RNA molecule of about $9.6 \mathrm{~Kb}$. It consists of large polyprotein of about 3000 amino acids. this protein consists of structural proteins; the core and the envelope, besides non-structural ones such as: NS3, NS4A, NS4B, NS5A and NS5B. These $\mathrm{HCV}$ proteins have several cellular functions besides their major function in viral replication (3). Accumulation of mutations during HCV replication leads to its significant genetic heterogeneity. The most heterogeneous parts of the genome are envelope proteins (E1 and E2) while the most conserved regions are parts of the 5'UTR and the terminal 3'UTR, while the core region was semi conserved (4). It had been found that both core and envelope proteins play a major role in various stages of the of the virus' life cycle: including cell entry, uncoating and virion assembly suggested that the core region with envelope proteins have numerous functional activities. These regions were found to play an important role in viral persistence by escaping from the the host's immune system (5). Hepatitis C virus (HCV) E1 and E2 glycoproteins had a high degree of variability which made the virus gained different phenotypic traits; as do alterations in receptorbinding affinity and in immune recognition and escape. It was found that HVR1 had high genetic diversity especially in patients with persistent viremia. Moreover, the rate of non-synonymous substitutions were predominated within the HVR1 region and gradually increased, compared to that in the E1 and E2 regions outside HVR1 (6).

Implication for health policy/practice/research/medical education:

Studying different spectra of viral strains of HCV 4 is considered an important research tool and as well as an important part of the diagnostic work up in clinical practice for the development of vaccine trials in Egypt and would help in explaining the existing intra-individual variations of patients in response to the existing drugs in the local market.

Copyright ( ) 2013, KowsarCorp.; Licensee Kowsar Ltd. This is an Open Access article distributed under the terms of the Creative Commons Attribution License (http:/ creativecommons.org/licenses/by/3.0), which permits unrestricted use, distribution, and reproduction in any medium, provided the original work is properly cited. 


\section{Objectives}

Disease course and antiviral therapy response are affected by a multiplicity of host and viral factors that are under extensive research worldwide. Certain viral genotypes, subtypes and quasispecies are linked to disease progression, however, other studies showed the contrary, thus, due to the heterogeneity of HCV with both high replication and mutation rate in vivo, this study was conducted to analyze different isolates of Egyptian patients in the most mutable (mutation prone) regions of the virus (E1 and E2). These data would be fundamental for the development of different trials for vaccine in Egypt.

\section{Patients and Methods}

\subsection{Patients}

This study was done on Egyptian patients infected with Hepatitis C virus (HCV). This work obeys ethical guidelines and an informed consent was obtained from each patient incorporated in the study. Patients in this study were subjected to the following:

Full history taking, clinical and laboratory examinations where the mean \pm SD of age was $(47.5 \pm 5.6)$ years old. Males percentage in this study was (80\%) while females were (20\%). An elevation in liver enzymes (ALT and AST) was observed in $75 \%$ of the cases studied.

\subsection{HCV RNA Tests}

These include qualitative HCV nested RT-PCR, genotyping of HCV RNA genome. Methods used for these assays were previously described as follows; nested RT-PCR (7) and HCV genotyping (8).

\subsection{Nested PCR Amplification of HCVE1-E2 Regions Using Specific Primers}

Primers' sequences were as follows: F1: 5' CACTGGACYACBCARGA NTGYAA 3'

(Where $\mathrm{Y}$ is $\mathrm{C}$ or $\mathrm{T}$; $\mathrm{B}$ is $\mathrm{G}, \mathrm{T}$, or $\mathrm{C}$; $\mathrm{R}$ is $\mathrm{G}$ or $\mathrm{A}$; and $\mathrm{N}$ is $\mathrm{A}, \mathrm{C}$, $\mathrm{G}$, or T)

F2: 5' ATGGCNTGGGAYATGATGATGAA 3'

(Where $\mathrm{N}$ is $\mathrm{A}, \mathrm{C}, \mathrm{G}$, or $\mathrm{T}$; and $\mathrm{Y}$ is $\mathrm{C}$ or $\mathrm{T}$ )

R1: 5' TTGGTGAACCCDGTRCYRTTCA 3'

(Where D is G, A, or T; R is G or A; and Y is C or T)

R2: 5' TGAACCCDGTRCYRTTCATTCA 3'

(Where D is G, A, or T; R is G or A; and Y is C or T). R3: 5' AGGAAGACATCNGTNTCRTTCTC 3'

(Where $\mathrm{N}$ is $\mathrm{A}, \mathrm{C}, \mathrm{G}$, or $\mathrm{T}$ and $\mathrm{R}$ is $\mathrm{G}$ or $\mathrm{A}$ )

cDNA synthesis and first round PCR were performed simultaneously using RT-PCR beads (Amersham Biosciences, USA), for each sample $6 \mu$ l of RNA were added with $5 \mu$ of each of the following specific primers (R1, R2 and F1). The reverse transcription Thermal cycling was 30 minutes at $42^{\circ} \mathrm{C}$ followed by 5 minutes at $94^{\circ} \mathrm{C}$. First PCR round was: 40 cycles for 40 seconds at $94^{\circ} \mathrm{C}$, 20seconds at $53^{\circ} \mathrm{C}$ and 1 minute at $72^{\circ} \mathrm{C}$, then final extension for $7 \mathrm{~min}$ utes at $72^{\circ} \mathrm{C}$. For the second amplification specific primers (F2 and R3) were used. The Thermal cycling protocol was 9 minutes at $95^{\circ} \mathrm{C}$ then 35 cycles for 40 seconds at $94^{\circ} \mathrm{C}, 10$ seconds at $53.5^{\circ} \mathrm{C}$ and 1 minute at $72^{\circ} \mathrm{C}$, then final extension for 7 minute at $72^{\circ} \mathrm{C} .10 \mu$ l of the each amplicon were electrophoresed on a $2 \%$ agarose gel.

\subsection{Cloning of DNA Fragments}

The pGEM-T Vector system (Promega, USA) was used to clone purified PCR products. The ligated plasmids were transformed into highly efficient competent cells namely JM 109. Plasmid DNA was isolated using plasmid miniprep method using (Wizard plus Minipreps DNA Purification System Kit, Promega-USA).

\subsection{Sequencing and Phylogenetic Analysis}

Samples further underwent sequence analysis in the Automated Sequencer “ABI Prism 310 Genetic Analyzer”. The sequences were aligned with the consensus sequences of genotype 4 that were retrieved from Gene Bank using the program ClustalX implemented in the Bioedit package. The PHYLIP suit of programs, version 3.572 (9) was used to generate the Phylogenetic tree.

The DNADIST program was used to calculate the Genetic distance matrices which were then used to generate a tree using NEIGHBOR and the SEQBOOT program. Reference sequences of different HCV genotypes were retrieved from Gene Bank.

\subsection{Computer-Assisted Analysis of N Glycosylation Sites and Epitope Prediction}

$\mathrm{N}$ glycosylation sites were predicted using the on-line prediction server NetNGlyc version 1.0.Epitopes. Prediction of T-cell was performed using the on-line software SYFPEITHI (version 1) (10).

\subsection{Secondary Structural Prediction}

All the E1/E2 sequence of 28 Egyptian clones was uploaded to the internet server, and the secondary structures waere predicted automatically using SOPMA library.

\section{Results}

\subsection{Clinical Data}

All the Egyptian patients infected with Hepatitis C Virus (HCV) fulfilled the criteria for being covered by the national health program for treating viral hepatitis and they were free from co-infection with HBV, HIV or Schistosomiases, no thyroid dysfunction, no uncontrolled diabetes mellitus, no obesity, no history of long term drug 
or alcohol intake.

\subsection{Sequencing and Phylogenetic Analysis}

Nucleotide and amino acid (a.a) sequences of the amplified HCV E1/E2 28 Egyptians clones were determined and aligned with sequences of HCV prototypes and other genotypes taken from Gene Bank.

The phylogenetic tree was generated using the reference sequences of HCV prototype (HCp) and genotypes of $\operatorname{HCV}(1 \mathrm{a}, 1 \mathrm{~b}, 2 \mathrm{a}, 2 \mathrm{~b}, 3 \mathrm{a}, 3 \mathrm{~b}, 4 \mathrm{a}, 4 \mathrm{c}, 4 \mathrm{f}, 5 \mathrm{a}$ and $6 \mathrm{a})$ retrieved from the Gene bank and the sequence data of the 28 Egyptian clones whose accession number in the Gene Bank is shown in Figure 1.

Figure 1. Phylogenetic Tree of HCV E1-E2 Regions of 28 Egyptian Strains

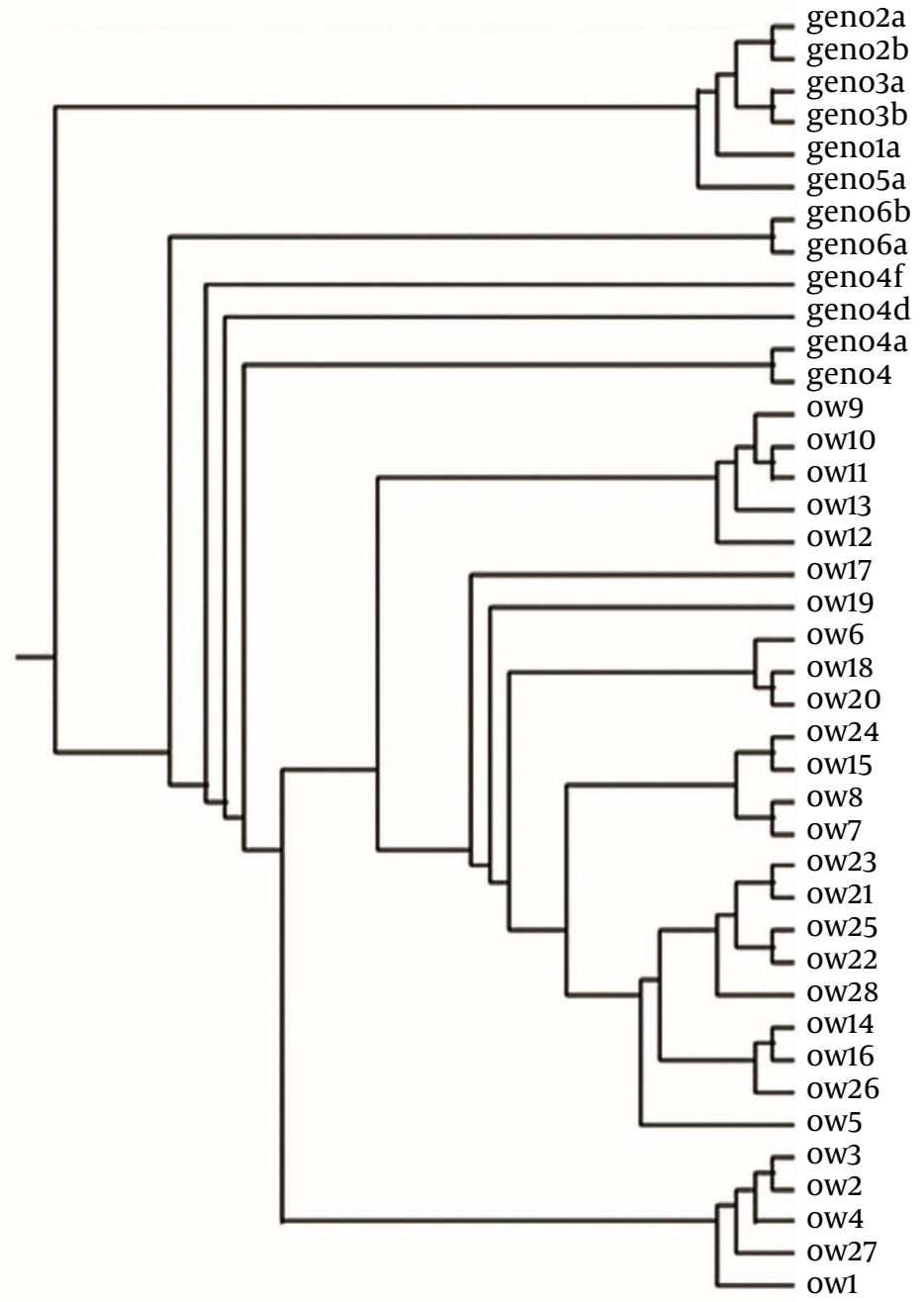

The sequence of HCV E1/E2 Egyptian clones unraveled in this study had been submitted to Gene Bank under the following accession numbers: OW1(HM596343), OW2(HM596344), OW3(HM596345), OW4(HM596346), OW5(HM596347), OW6(HM749053), OW7(HM596348), OW8(HM596349), OW9(HM596350), OW10(HM596351),OW11(HM596352), OW12 (HM623431), OW13 (HM623432), OW14 (HM623433), OW15 (HM623434),OW16 (HM623435), OW17 (HM623436), OW18 (HM623437), OW19(HM623438), OW20 (HM623439),OW21(HM623440), OW22(HM623441), OW23 (HM749054), OW24 (HM749055), OW25 (HM749056),OW26 (HM749057), OW27 (HM749058), OW28 (HM749059).

\subsection{Multiple Alignment}

Multiple alignment of E1/E2 amino acid sequences of 28 Egyptian local strains with prototype 4 a and genotype 4 is shown in Figure 2 and in HCV E1/ E2 Domains for HVR1 (aa384-410) (Figure 3), HVR2 (aa474-482) and CD81-1(474494) (Figure 4) and CD81-2(aa522-551) (Figure 5). 
Ow1

OW2 7

OW2

OW3

OW2 6

OW28

(1)

OW22

OW19

OW16

OW8

OW7

OW6

OW18

OW20

OW1 4

OW15

OW24

OW5

OW1 7

OW21

OW23

OW9

OW10

OW11

OW13

OW12

geno 4 a

geno 4 MAWDMMMNWS PTTTLVLAQIMRI PGTLVDLFAGGHWGVLVGVAYFCMOANWAKVILVLFI MAWDMMMNWSPTTTLVLAQIMRI PGTLVDLFAGGHWGVLVGVAYFCTOANWAKVILVLFI MAWDMMMNWSPTTTLVLAOIMRI PGTLVDLFAGGHWGVLVGVAYFCTOANWAKVILVLFI MAWDMMMNWSPTTTLVLAQIMRI PGTLVDLFAGGHWGVLVGVAYFCMQANWAKVIL ILFL MAWDMMMNWSPTTTLVLAQIMRI PGTLVDLLAGGHWGVLVGVAYFCMQANWAKVILVLFI MAWDMMMNWS PTTTLVLAOIMRI PGTLVDLLAGGHWGVLVGVAYFCMOANWAKVILVLFL MAWDMMMNWSPTTTLVIAAOMRI PGTLVDLLAGGHWGVLVGVAYFCMOANWAKVILVLFI MAWDMMMNWSPTTTLVLAQIMRI PGTLVDLLAGGHWGVLVGVAYFCMQANWAKVILVLFL MAWDMMMNWSPTTTLVLAQIMRI PGTLVDLLAGGHWGVLVGVAYFCMQANWAKVILVLFL MAWDMMMNWS PTTTLVLAOIMRI PGTLVDLLAGGHWGVLVGVAYFCMOANWAKVILVLFL MAWDMMMNWSPTTTLVLAOIMRI PGTLVDLLAGGHWGVLVGVAYFCMOANWAKVILVLFL MAWDMMMNWS PTTTLVLAQIMRI PGTLVDLLAGGHWGVLVGVAYFCMQANWAKVILVLFL MAWDMMMNWSPTTTLVLAQIMRI PGTLVDLLAGGHWGVLVGVAY FCMQANWAKVILVLFL MAWDMMMNWS PTTTLVLAOIMRI PGTLVDLLAGGHWGVLVGVAYFCMOANWAKVILVLFL MAWDMMMNWS PTTTLVIAOIMRI PGTLVDLLAGGHWGVLVGVAYFCMOANWAKVILVLFL MAWDMMMNWS PTTTLVLAQIMRI PGTLVDLLAGGHWGVLVGVAYFCMQANWAKVVLVLFL MAWDMMMNWSPTTTLVLAQIMRI PGTLVDLLAGGHWGVLVGVAYFCMQANWAKVVLVLFL MAWDMMMNWSPTTTLVLAOIMRI PGTLVDLLAGGHWGVLVGVAYFCMOANWAKVILVLFL MAWDMMMNWSPTTTLVLAOIMRI PGTLVDLLAGGHWGVLEEVAY FCMOANWAKVIL I LFL MAWDMMMNWSPTTALVLAQIMRI PGTLVDLLAGGHWGVLVGVAYFCMQANWAKVILVLFL MAWDMMMNWS PTTTLVLAQIMRI PGTLVDLLAGGHWGVLVGVAYFCMQANWAKVIIVLFL MAWDMMMNWSPTTTLVLAOIMRI PGTLVDLLAGGHWGVLVGVAYFCMOANWAKVILVLFL MAWDMMMNWSPTTTLVLAOIMRI PGTLVDLLAGGHWGVLVGVAYFCMOANWAKVILVLFL MAWDMMMNWS PTTTLVLAQIMRI PGTLVDLLAGGHWGVLVGVAYFCMQANWAKVILVLFL MAWDMMMNWS PTTTLVLAQIMRI PGTLVDLLAGGHWGVLVGVAYFCMQANWAKVIIVLFL MAWDMMMNWSPTTTLVLAQIMRI PGTLVDLLAGGHWGVLVGVAYFCMOANWAKVILVLFL MAWDMMMNWS PTTTLVLAOIMRI PGTLVDLLAGGHWGVLVGVAYFCMOANWAKVILVLFL MAWDMMMNWSPTTTLVLAQVMRI PTTLVDLLSGGHWGVLVGVAYFSMQANWAKVILVLFL MAWDMMMNWSPTTTLVISQVMRI P S ILVDLLAGGHWGVLVGVAYFSMQANWAKVIXVLFL

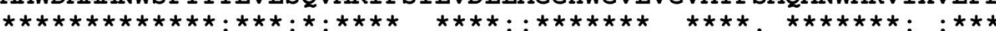

Figure 2. Multiple Alignment for E1/E2 Amino Acid Sequences Means that all the sequences are identical in in the alignment. showed the conserved substitutions. "showed the semi-conserved substitutions. The space means that substitutions are observed.

OW1

OW2 7

OW2

OW3

OW 4

OW2 6

OW28

OW25

OW22

OW19

OW1 6

OW8

OW7

OW6

OW18

OW20

OW14

OW1

OW1

OW24

ow5

OW17

OW21

OW23

ow9

OW10

OW11

OW13

OW13

OW12

geno 4

HVR1 (aa384-410)

FAGVDANTHVTGAVAGHTAAGFAGLFSPGAQQRIQLVNKNGSWH INRTALNCNDSLNTGF FAGVDANTHVTGAVAGHTAAGFAGLFSP GAQQRIQLVNKNGSWH INRTALNCNDSLNTGF FAGVDANTHVT GAVA GHTAA GFA GLFS P GAQQRIQLVNKNGSWH INRTALNCNDSLNTGF FAGVDANTHVT GAVAGHTAAGFAGLFSPGAOORIOLVNKNGSWH INRTALNCNDSLNTGF FAGVDANTHVT GAVAGHTAAGFAGLFSPGAQQRIQLVNKNGSWH INRTALNCNDSLNTGF FAGVDANTHVT GAMA GQQAASLAS LFTP GAQQRIQLVNKNGSWH INRTALNCND SLNTGF FAGVDANTHVT GAMAGQQAAS LAS LFTP GAQQRIQLVNKNGSWH INRTALNCNDSLNTGF FAGVDANTHVT GAMAGOOAASTAS LFTPGAOORIOLVNKNGSWH INRTALNCNDSLNTGF FAGVDANTHVT GAMAGOOAASIAAS LFT PGAOORIOLVNKNGSWH INRTALNCNDSLNTGF FAGVDANTHVT GAMAGQQAAS LAS LFTP GAQQRIQLVNKNGSWH INRTALNCNDSLNTGF FAGVDANTHVT GAMA GQQAASLASLFTP GAQQRIQLVNKNGSWH INRTALNCNDSLNTGF FAGVDANTHVT GAMA GQQAAS LAS LFT PGAQQRIQLVNKNGSWH INRTALNCNDSLNTGF FAGVDANTHVT GAMA GOOAASTAS LFTPGAOORIOLVNKNGSWH INRTALNCND SLNTGF FAGVDANTHVT GAMAGQQAAS LAS LFT PGAQQRIQLVNKNGSWH INRTALNCNDSLNTGF FAGVDANTHVT GAMA GQQAASLAS LFTP GAQQRIQLVNKNGSWH INRTALNCNDSLNTGF FAGVDANTHVT GAMAGQQAASLAS LFTP GAQQRIQLVNKNGSWH INRTALNCNDSLNTGF FAGVDANTHVTGAMAGQQAASLASLFTPGAQQRIQLVNKNGSWH INRTALNSNDRLNTGF FAGVDANTHVTGAMAGOOAAST,ASTFTPGAOORIOLVNKNGSWH INRTALNCNDSLNTGF FAGVDANTHVT GAMAGQQAASLASLFTPGAQQRIQLVNKNGSWH INRTALNCNDSLNTGF FAGVDANTHVT GAMAGQQAAS LAS LFTP GAQQRIQLVNKNGSWH INRTALNCNDSLNTGF FAGVDANTHVT GAMAGQQAASLAGLFTP GAQQRMQLVNKNGSWH INRTALNCNDSLNTGF FAGVDANTHVT GAMAGQQAASLAS LFTPGAQQRIQLVNKNGSWHINRTALNCNDSLNTGF FAGVDANTHVT GAMAGQQAASLAS LFTPGAQORIQLVNKNGSWH INRTALNCNDSLNTGF FAGVDANTHVT GAMAGQQAAS LAS LFTPGAQQRIQLVNKNGSWH INRTALNCNDSLNTGF FAGVDANTHVT GAMA GQQAASLAS LFTP GAQQRIQLVNKNGSWH INRTALNCNDSLNTGF FAGVDANTHVT GAMAGOOAASIASIFTPGAOORIOLVNKNGSWHINRTALNCNDSLNTGF FAGVDANTHVT GAMAGOOAASTAASIFTPGAOORIOLVNKNGSWH INRTALNCNDSLNTGF FAGVDANTHVT GAMAGQQAASLASLFTPGAQQRIQLVNKNGSWH INRTALNCNDSLNTGF FAGVDAETHVS GAAVGRSTAGLANLFSSGSKQNLQL INSNGSWH INRTALNCNDSLNTGF FAGVDARTYVSGGVTSXTTGGLVSLISXGARQNLQLINSNGSWHINRTALNCNDSLNTGF

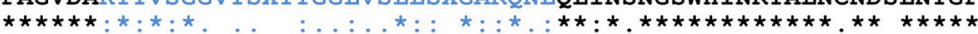

Figure 3. Multiple Alignment for E1-E2 (HVR1) Amino Acids of 28 Egyptian Local Strains with Prototype 4a Means that all the sequences are identical in in the alignment. showed the conserved substitutions. "showed the semi-conserved substitutions. The space means that substitutions are observed. 
Omran MH et al.

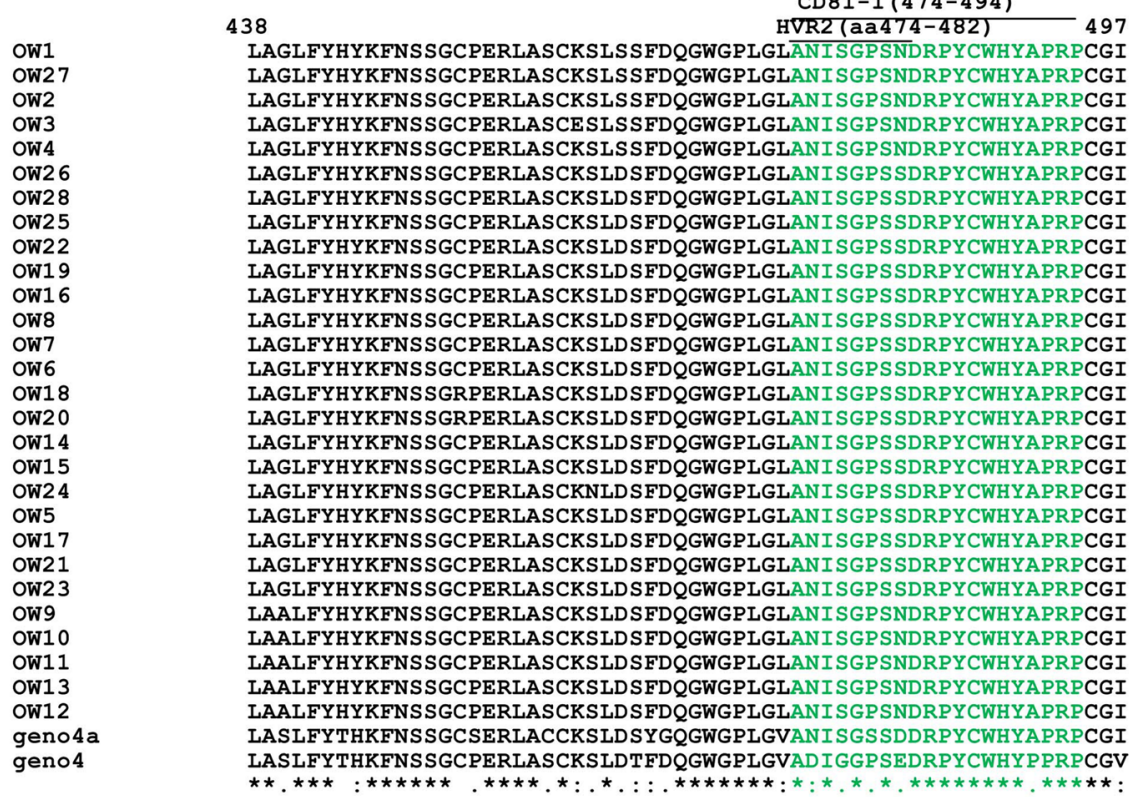

Figure 4. Multiple Alignment for E1-E2 (HVR2 and CD81-1) Amino Acids of 28 Egyptian local strains with prototype 4a "Means that all the sequences are identical in in the alignment. "showed the conserved substitutions. "showed the semi-conserved substitutions. The space means that substitutions are observed.

CD81-2(aa522-551)

538

OW1

OW27

ow2

ow3

OW4

OW2 6

OW28

Ow25

OW22

OW19

OW1 6

OW8

ow7

OW6

OW18

OW20

OW14

OW15

OW2 4

OW5

OW17

OW21

ow23

OW9

OW10

OW11

OW13

OW12

Pro. 4 a

geno 4

\begin{abstract}
VPAASVCGPVYCFTPSPVVVGTTDRFGVPTYTWGENDTDVF VPAASVCGPVYCFTPSPVVVGTTDRFGVPTYTWGENDTDVF VPAASVCGPVYCFTPSPVVVGTTDRFGVPTYTWGENETDVF VPAASVCGPVYCFTPSPVVVGTTDRFGVPTYTWGENDTDVF VPAASVCGPVYCFTPSPVVVGTTDRFGVPTYTWGENETDVF VPAS SVCGPVYCFTPSPVVVGTTDHFGVPTYTWGENDTDVF VPASSVCGPVYCFTPSPVVVGTTDHFGVPTYTWGENDTDVF VPASSVCGPVYCFTPSPVVVGTTDHFGVPTYTWGENDTDVF VPASSVCGPVYCFTPSPVVVGTTDHFGVPTYTWGENDTDVF VPASSVCGPVYCFTPSPVVVGTTDHFGVPTYTWGENDTDVF VPAS SVCGPVYCFTPSPVVVGTTDHFGVPTYTWGENDTDVF VPAS SVCGPVYCFTPSPVVVGTTDHFGVPTYTWGENDTDVF VPASSVCGPVYCFTPSPVVVGTTDHFGVPTYTWGENDTDVF VPASSVCGPVYCFTPSPVVVGTTDHFGVPTYTWGENDTDVF VPAS SVCGPVYCFTPSPVVVGTTDHFGVPTYTWGENDTDVF VPASSVCGPVYCFTPSPVVVGTTDHFGAPTYTWGENDTDVF VPAS SVCGPVYCFTPSPVVVGTTDHFGVPTYTWGENDTDVF VPASSVCGPVYCFTPSPVVVGTTDHFGVPTYTWGENDTDVF VPASSVCGPVYCFTPSPVVVGTTDHFGVPTYTWGENDTDVF VPAS SVCGPVYCFTPSPVVVGTTDHFGVPTYTWGENDTDVF VPASSVCGPVYCFTPSPVVVGTTDHFGVPTYTWGENDTDVF VPAS SVCGPVYCFTPSPVVVGTTDHFGVPTYTWGENETDVF VPAS SVCGPVYCFTPSPVVVGTTDHFGVPTYTWGENETDVF MPAS SVCGPVYCFTPS PVVVGTTDHFGVPTYTWGENETDVF MPASSVCGPVYCFTPSPVVVGTTDHFGVPTYTLGGNETDVF MPASSVCGPVYCFTPSPVVVGTTDHFGVPTYTWGENETDVF MPASSVCGPVYCFTPSPVVVGTTDHFGVPTYTWGENETDVF MPASSVCGPVYCFTPSPVVVGTTDHFGVPTYTWGENDTDVF VPASSVCGPVYCFTPSPVVVGTTDHVGVPTYTWGENETDVF VPARSVCGPVYCFTPSPVVVGTTDRLGVPTYTWGENE SDVF

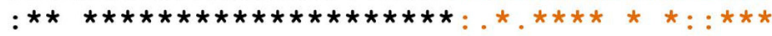

Figure 5. Multiple Alignment for E1-E2 (CD81-2) Amino Acids of 28 Egyptian Local Strains With Prototype 4a "Means that all the sequences are identical in in the alignment." showed the conserved substitutions. "showed the semi-conserved substitutions. The space means that substitutions are observed. 


\subsection{Probability of Glycosylation Sites}

In the present study, all of the isolates showed four different N-glycosylation sites, except for OW14 (HM623433) which showed only three sites. Potential glycosylation sites verge at positions $100,113,159$, and 216 . The probability of the potential glycosylation sites in the 28 Egyptian strains are shown in (Table 1), glycosylation sites at positions $100,113,159$, and 216 correspond to amino acids 418 , 431, 477 and 534 in HCV 4a.

\begin{tabular}{|c|c|c|c|c|c|c|c|c|}
\hline \multirow[t]{2}{*}{ Isolate } & \multicolumn{7}{|c|}{ Probability of glycosylation position } & \multirow[t]{2}{*}{ No. of sites } \\
\hline & 8 & 100 & 106 & 113 & 131 & 159 & 216 & \\
\hline OW1 & -- & $+^{\mathrm{a}}$ & -a & + & - & + & + & 4 \\
\hline OW2 & -- & + & - & + & - & + & + & 4 \\
\hline OW3 & -- & + & - & + & - & + & + & 4 \\
\hline OW4 & -- & + & - & + & - & + & + & 4 \\
\hline OW5 & -- & + & - & + & - & + & + & 4 \\
\hline OW6 & -- & + & - & + & - & + & + & 4 \\
\hline OW7 & -- & + & - & + & - & + & + & 4 \\
\hline OW8 & -- & + & - & + & - & + & + & 4 \\
\hline OW9 & - & + & - & + & - & + & + & 4 \\
\hline OW10 & - & + & - & + & - & + & + & 4 \\
\hline OW11 & - & + & - & + & - & + & + & 4 \\
\hline OW12 & - & + & - & + & - & + & + & 4 \\
\hline OW13 & - & + & - & + & - & + & + & 4 \\
\hline OW14 & - & + & - & $\begin{array}{l}\text { Not pre- } \\
\text { dicted }\end{array}$ & - & + & + & 3 \\
\hline OW15 & -- & + & - & + & - & + & + & 4 \\
\hline OW16 & -- & + & - & + & - & + & + & 4 \\
\hline OW17 & -- & + & - & + & - & + & + & 4 \\
\hline OW18 & -- & + & - & + & - & + & + & 4 \\
\hline OW19 & -- & + & - & + & - & + & + & 4 \\
\hline OW20 & -- & + & - & + & - & + & + & 4 \\
\hline OW21 & -- & + & - & + & - & + & + & 4 \\
\hline OW22 & - & + & - & + & - & + & + & 4 \\
\hline OW23 & - & + & - & + & - & + & + & 4 \\
\hline OW24 & - & + & - & + & - & + & + & 4 \\
\hline OW25 & - & + & - & + & - & + & + & 4 \\
\hline OW26 & - & + & - & + & - & + & + & 4 \\
\hline OW27 & - & + & - & + & - & + & + & 4 \\
\hline OW28 & - & + & - & + & - & + & + & 4 \\
\hline Geno 4a & -- & ++ & - & + & - & + & + & 4 \\
\hline
\end{tabular}

$\mathrm{a}_{+, \text {Probability of glycosylation sites; - , Glycosylation sites not present }}$

4.5. Synonymous and Non-Synonymous Substitution Rates

Calculation of synonymous and non-synonymous sub- stitution rates in 28 Egyptians clones compared to prototype 4a (Y11604) are shown in (Table 2). 
Omran MH et al.

\begin{tabular}{|c|c|c|c|c|c|c|c|c|c|c|c|c|c|}
\hline \multicolumn{2}{|c|}{ Compare } & \multirow{2}{*}{$\begin{array}{l}\text { Sequences } \\
\text { Y11604(4a) }\end{array}$} & \multirow{2}{*}{$\begin{array}{l}\text { names } \\
\text { OW1 }\end{array}$} & \multirow{2}{*}{$\begin{array}{l}\mathbf{S d}^{\mathrm{a}} \\
58.0000\end{array}$} & \multirow{2}{*}{$\frac{S^{a}}{44.0000}$} & \multirow{2}{*}{$\frac{S^{a}}{152.1667}$} & \multirow{2}{*}{$\begin{array}{l}\text { No. }^{\mathrm{a}} \\
513.8333\end{array}$} & \multirow{2}{*}{$\frac{\mathbf{P s}^{\mathrm{a}}}{0.3812}$} & \multirow{2}{*}{$\frac{\mathbf{P n}^{\mathrm{a}}}{0.0856}$} & \multirow{2}{*}{$\begin{array}{l}\text { Ds }^{\mathrm{a}} \\
0.5323\end{array}$} & \multirow{2}{*}{$\frac{\text { Dn }^{\mathrm{a}}}{0.0909}$} & \multirow{2}{*}{$\begin{array}{l}\mathbf{d s} / \mathbf{d n}^{\mathrm{a}} \\
5.8540\end{array}$} & \multirow{2}{*}{$\begin{array}{c}\text { ps/pn } \\
4.4512\end{array}$} \\
\hline $\mathbf{0}$ & 1 & & & & & & & & & & & & \\
\hline $\mathbf{0}$ & 2 & Y11604 (4a) & OW2 & 56.0000 & 44.0000 & 152.1667 & 513.3333 & 0.3668 & 0.0857 & 0.5037 & 0.0910 & 5.5335 & 4.2935 \\
\hline $\mathbf{0}$ & 3 & Y11604 (4a) & OW3 & 56.0000 & 46.0000 & 152.1667 & 513.3333 & 0.3668 & 0.0896 & 0.5037 & 0.0954 & 5.2777 & 4.0934 \\
\hline $\mathbf{0}$ & 4 & Y11604 (4a) & OW4 & 57.0000 & 44.0000 & 152.0000 & 514.0000 & 0.3750 & 0.0856 & 0.5199 & 0.0909 & 5.7193 & 4.3807 \\
\hline $\mathbf{0}$ & 5 & Y11604 (4a) & OW5 & 49.0000 & 46.0000 & 149.6667 & 516.3333 & 0.3274 & 0.0891 & 0.4302 & 0.0948 & 4.5363 & 3.6749 \\
\hline $\mathbf{0}$ & 6 & Y11604 (4a) & OW6 & 48.0000 & 43.0000 & 150.5000 & 515.5000 & 0.3189 & 0.0834 & 0.4154 & 0.0884 & 4.6972 & 3.8235 \\
\hline $\mathbf{0}$ & 7 & Y11604 (4a) & OW7 & 48.0000 & 43.0000 & 150.5000 & 515.5000 & 0.3189 & 0.0834 & 0.4154 & 0.0884 & 4.6972 & 3.8235 \\
\hline $\mathbf{0}$ & 8 & Y11604 (4a) & OW8 & 48.0000 & 43.0000 & 150.5000 & 515.5000 & 0.3189 & 0.0834 & 0.4154 & 0.0884 & 4.6972 & 3.8235 \\
\hline $\mathbf{0}$ & 9 & Y11604 (4a) & OW9 & 53.0000 & 43.0000 & 150.5000 & 516.0000 & 0.3533 & 0.0833 & 0.4777 & 0.0883 & 5.4081 & 4.2259 \\
\hline $\mathbf{0}$ & 10 & Y11604 (4a) & OW10 & 56.0000 & 45.0000 & 150.6667 & 515.3333 & 0.3717 & 0.0873 & 0.5133 & 0.0928 & 5.5285 & 4.2564 \\
\hline $\mathbf{0}$ & 11 & Y11604 (4a) & OW11 & 54.0000 & 43.0000 & 150.0000 & 516.0000 & 0.3600 & 0.0833 & 0.4904 & 0.0883 & 5.5395 & 4.3200 \\
\hline $\mathbf{0}$ & 12 & Y11604 (4a) & OW12 & 55.0000 & 44.0000 & 150.3333 & 515.6667 & 0.3659 & 0.0853 & 0.5018 & 0.0906 & 5.5395 & 4.2877 \\
\hline $\mathbf{0}$ & 13 & Y11604 (4a) & OW13 & 51.0000 & 43.0000 & 150.0000 & 516.0000 & 0.3400 & 0.0833 & 0.4529 & 0.0883 & 5.1274 & 4.0800 \\
\hline $\mathbf{0}$ & 14 & Y11604 (4a) & OW14 & 48.0000 & 46.0000 & 150.8333 & 515.1667 & 0.3182 & 0.0893 & 0.4141 & 0.0951 & 4.3561 & 3.5640 \\
\hline $\mathbf{0}$ & 15 & Y11604 (4a) & OW15 & 48.0000 & 44.0000 & 150.6667 & 515.3333 & 0.3186 & 0.0854 & 0.4148 & 0.0906 & 4.5755 & 3.7313 \\
\hline $\mathbf{0}$ & 16 & Y11604 (4a) & OW16 & 51.0000 & 43.0000 & 150.5000 & 515.5000 & 0.3389 & 0.0834 & 0.4509 & 0.0884 & 5.0988 & 4.0625 \\
\hline $\mathbf{0}$ & 17 & Y11604 (4a) & OW17 & 49.5000 & 45.5000 & 150.5000 & 515.5000 & 0.3289 & 0.0883 & 0.4329 & 0.0939 & 4.6101 & 3.7264 \\
\hline $\mathbf{0}$ & 18 & Y11604 (4a) & OW18 & 51.0000 & 44.0000 & 150.8333 & 515.1667 & 0.3381 & 0.0854 & 0.4495 & 0.0907 & 4.9573 & 3.9588 \\
\hline $\mathbf{0}$ & 19 & Y11604 (4a) & OW19 & 51.0000 & 43.0000 & 150.5000 & 515.5000 & 0.3389 & 0.0834 & 0.4509 & 0.0884 & 5.0988 & 4.0625 \\
\hline o & 20 & Y11604 (4a) & OW20 & 53.0000 & 45.0000 & 150.8333 & 515.1667 & 0.3514 & 0.0874 & 0.4741 & 0.0929 & 5.1044 & 4.0227 \\
\hline $\mathbf{0}$ & 21 & Y11604 (4a) & OW21 & 49.0000 & 42.0000 & 150.5000 & 515.5000 & 0.3256 & 0.0815 & 0.4270 & 0.0862 & 4.9510 & 3.9961 \\
\hline $\mathbf{0}$ & 22 & Y11604 (4a) & OW22 & 48.0000 & 43.0000 & 150.5000 & 515.5000 & 0.3189 & 0.0834 & 0.4154 & 0.0884 & 4.6972 & 3.8235 \\
\hline $\mathbf{0}$ & 23 & Y11604 (4a) & OW23 & 49.0000 & 42.0000 & 150.5000 & 515.5000 & 0.3256 & 0.0815 & 0.4270 & 0.0862 & 4.9510 & 3.9961 \\
\hline $\mathbf{0}$ & 24 & Y11604 (4a) & OW24 & 50.0000 & 44.0000 & 150.8333 & 515.1667 & 0.3315 & 0.0854 & 0.4375 & 0.0907 & 4.8252 & 3.8812 \\
\hline $\mathbf{0}$ & 25 & Y11604 (4a) & OW25 & 48.0000 & 43.0000 & 150.5000 & 515.5000 & 0.3189 & 0.0834 & 0.4154 & 0.0884 & 4.6972 & 3.8235 \\
\hline $\mathbf{0}$ & 26 & Y11604 (4a) & OW26 & 50.0000 & 43.0000 & 150.5000 & 515.5000 & 0.3322 & 0.0834 & 0.4388 & 0.0884 & 4.9628 & 3.9828 \\
\hline $\mathbf{0}$ & 27 & Y11604 (4a) & OW27 & 53.0000 & 44.0000 & 152.1667 & 513.8333 & 0.3483 & 0.0856 & 0.4683 & 0.0909 & 5.1501 & 4.0675 \\
\hline $\mathbf{0}$ & 28 & Y11604 (4a) & OW28 & 50.0000 & 43.0000 & 150.5000 & 515.5000 & 0.3322 & 0.0834 & 0.4388 & 0.0884 & 4.9628 & 3.9828 \\
\hline
\end{tabular}




\subsection{Prediction of the Epitope Domain}

Six classes of epitopes were observed in the 28 Egyptian strains, first class was (H2-Db nonamers), second class (H2-Kd nonamer), third class (HLA-A*26), fourth class (HLA-B*1402), fifth class (HLA-B*2705) and sixth class (HLA-A*0201). The prediction of the epitope domains in E1-E2 regions of 28 Egyptian strains was shown in Table 3.

\begin{tabular}{|c|c|c|c|}
\hline Score & Epitope Domains of Egpytian stains & Position $^{\mathrm{a}}$ & Epitope Domains classes \\
\hline & OW1.....OW28 & & $\mathrm{H} 2-\mathrm{Db}$ \\
\hline & $4 \mathrm{a}$ & & Nonamer \\
\hline \multirow[t]{3}{*}{24} & SWHINRTAL & 420 & \\
\hline & OW1.....OW28 & & $\mathrm{H} 2-\mathrm{kD}$ \\
\hline & $4 \mathrm{a}$ & & Nonamer \\
\hline \multirow[t]{4}{*}{24} & HYAPRPCG I & 490 & \\
\hline & OW1......WW3 & & HLA-A*26 \\
\hline & OW6.....OW28 & & Nonamer \\
\hline & $4 \mathrm{a}$ & & \\
\hline \multirow[t]{2}{*}{26} & KVILVLFLF & 371 & \\
\hline & OW4,OW5 & & \\
\hline \multirow[t]{3}{*}{28} & KVILILFLF & 371 & \\
\hline & OW1.....OW28 & & HLA-B 1402 \\
\hline & $4 \mathrm{a}$ & & Nonamer \\
\hline \multirow[t]{3}{*}{25} & E R L AS C K S L & 455 & \\
\hline & OW1.....OW23 & & HLA-B*2705 \\
\hline & OW25.....OW28 & & Nonamer \\
\hline \multirow[t]{2}{*}{24} & E R L AS C KS L & 455 & \\
\hline & OW24 & & \\
\hline \multirow[t]{2}{*}{25} & E R L AS C K N L & 455 & \\
\hline & $4 \mathrm{a}$ & & \\
\hline \multirow[t]{2}{*}{23} & E R LACCKSL & 455 & \\
\hline & OW1.....OW28 & & HLA-A*0201 \\
\hline \multirow[t]{2}{*}{26} & R I PG T L VD L & 340 & Nonamer \\
\hline & $4 a$ & & \\
\hline \multirow[t]{3}{*}{24} & RIPT TLVDL & 340 & \\
\hline & OW1......OW4 & & \\
\hline & OW27 & & \\
\hline \multirow[t]{3}{*}{19} & LFAGGHWGV & 348 & \\
\hline & OW5.....OW26 & & \\
\hline & OW28 & & \\
\hline \multirow[t]{2}{*}{29} & LLAGGHWGV & 348 & \\
\hline & $4 \mathrm{a}$ & & \\
\hline \multirow[t]{5}{*}{27} & L LSGGHWGV & 348 & \\
\hline & OW1.....OW3 & & \\
\hline & OW6......W9 & & \\
\hline & OW12.....OW28 & & \\
\hline & $4 a$ & & \\
\hline \multirow[t]{3}{*}{21} & LVLFLFAGV & 374 & \\
\hline & OW4,OW5 & & \\
\hline & OW10,.OW11 & & \\
\hline 26 & LI L F LFAGV & 374 & \\
\hline
\end{tabular}

\subsection{Secondary structural Results}

The data have showen differences between different HCV Egyptian isolates and the prototype $4 \mathrm{a}$ in the protein secondary structure.. In our isolates, 51 aa. of the 221 aa. scored an alpha helix secondary structures, whereas in the prototype 4a this score was observed for 47aa of the 221 aas. Extended strand (Ee) in our Egyptian isolates re- 
corded (60aa. up to 64aa.) while the prototype 4a recorded (56aa). Beta turn (Tt) in our Egyptian isolates recorded up to (17aa.) and in prototype 4 a recorded (13aa.) and random coil (Cc) in our Egyptian isolates recorded (91aa. up to 102aa.) and in prototype 4 a recorded 105aa.

\section{Discussions}

Egypt is considered the best model site for vaccine trials because the prevalence of HCV infections is high and extreme heterogeneity of HCV found at all levels. The development of an effective vaccine is influenced by many factors like proper folding of structural proteins, the eliciting of a neutralizing immune response and more importantly the viral heterogeneity due to its great role in disease progression therapy. This leads us to study in this research the most mutation prone regions of the virus (E1 and E2) in different isolates of Egyptian patients. Within the infected host a single isolate comprises many millions of both different and closely related sequences called quasispecies that provided a large store of different viral variants that had significant clinical effects helping the virus to escape the immune mechanism (11). The present work established that the heterogeneity of the quasispecies of our local strains of E1-E2 was high showing up to $15 \%$ diversity that might have important consequences during a transmission route depending on the number of transmitted viral RNA copies that might be limited and not representing the true complexity of the sequence diversity of the donor (12). However, this bottleneck could also be interpreted as selection of the optimal strain in the new host during the earliest infection events (13).

Accumulation of mutations during viral replication was either silent synonymous that had no effect on the sequence of the viral protein, but sometimes could affect the secondary structure of RNA. The other one was called non-synonymous mutations that caused changes in protein sequence that led to the production of different newly defective viral variants that could be lethal and aggressive $(14,15)$. The presented data in this research were interesting in light of analyzing the substitution rates of synonymous and non-synonymous mutations of the HCV E1-E2 local strains as the average number of potential synonymous substitutions in the sequences of the local isolates were 150.7 while those of the potential non-synonymous substitutions were 515 (Table 2).Thus, the number of non-synonymous mutations exceeded the number of synonymous mutations three times that warns us to the emergence of aggressive variants as these mutations led to the production of defective viral particles in the Egyptian population and could be lethal.

By having the sequencing data from the core gene of HCV genotype 4 of the Egyptian strains that had showed genetic relevance to HCV types 1 (16), in addition to the high number of the non-synonymous mutations of E1-E2 found in this study, suggests that the evaluation of the genotype 4 could happen in the same patient through multiple mutational ways of type 4 into genotype 1 which is closely related to it. Moreover, this might explain why the majority of the Egyptians, about 70\%, did not respond to interferon therapy. In accordance to (17) who reported that genetic variations of NS5 and E2 proteins of HCV genotype 1 would inhibit the double stranded (ds) RNAdependent protein kinase that is involved in the cellular antiviral response induced by interferon.

It was reported that N-linked glycans in viral envelope proteins could play a major role in the viral entry, or in modulating the response to treatment $(18,19)$. In the present study, all of the 28 Egyptian isolates showed four different glycosylation sites at positions 418, 431, 477 and 534 with the exception of clone OW14 (HM623433) which lacked the glycosylation site at position 431 (Table 1). Those glycans were found to play an important role in HCV entry and/or protein folding (20). In this study, two of the glycans $(477,534)$ were in the HVR2 and in the binding site of CD81 indicating that these regions were major targets for neutralizing antibodies. Furthermore, those glycans on E2 that attached to the binding site of CD81 might reduce the sense of HCV pp (HCV pseudo-particles) to antibody neutralization and consequently reduce the access of CD81 to its E2 binding site (21). On the other hand, (22) found that the absence of glycans at positions: $196,305,556$ or 623 strongly influences the incorporation of HCV glycoproteins into HCVpp (HCV pseudo-particles), suggesting that these glycans are essential for protein folding. This was long-established by our data, as it lacked these glycans, suggesting the effect of their absence in viral entry.

It was found that most of the induced antibodies had no antiviral activity. This was because they either targeted against useless epitopes that had no role in virus entry or they were produced by degraded intracellular proteins released from dying cells (23). Only "neutralizing antibodies" succeeded in both targeting the exposed epitopes and neutralizing the viral infection by controlling or preventing it (24). Also, it was known that identification of many CTL epitopes is required especially for the development of a successful immunogenic vaccine.

This work observed six classes of epitopes shown in the results section (Table 3 ). The present data showed that the class (HLA-A*0201) exhibits three epitopes (Table 3), two of them could be utilized as having score more than the prototype 4a. The first one at (aa 340-348) and the second one at (aa 348-356) where 23 clones had mutations of score 29 more than that prototype 4 a which is of score 27 indicating decreasing affinity of binding. However, (25) represented (HLA-A0201) an epitope that was not utilized because it did not have a proteasome cleavage site and had mutations that caused a decrease in the score. Also, two clones of the second class of epitope (HLA-A*26) (aa 371-379) had mutations and a score of 28 more than the prototype $4 \mathrm{a}$ and these could be used for synthetic HCV 
peptides. The fifth class (HLA-B*2705) (aa 455-463) has all the 28 Egyptian isolates had score of 24 higher than the score of prototype $4 \mathrm{a}$ (23) with the exception of clone (OW24 of score 25) (Table 3). These epitopes could be targeted by neutralizing antibodies and could exhibit broad cross-neutralizing activity among all major genotypes of HCV pp (HCV pseudo-particles) entry (26). Also, these epitopes could have a role in membrane fusion processes and could represent a target for monoclonal antibodies $(27,28)$. The present work also showed the sixth class (H2Kd nonamer) (aa 490-498) where all the 28 Egyptian isolates had score of 24 the same as the score of prototype 4a (Table 3) and those epitopes are different from that shown before in former report (25). Those epitopes could be essential for immunoreactivity as the binding site of E2 of CD81 located at aa480-493 and aa544-551 of E2 (29).

Furthermore, our data showed that the secondary structures of the E1-E2 region of our Egyptian HCV strains are different from former report (25). They have conformational and folding structure stronger than that of the prototype 4a. These secondary structures in this study might help the HCV polyprotein to undergo a series of cleavages to form functional viral proteins and guide HCV to hide their epitopes from the immune system and this coincides with former studies $(21,30)$.

The results of this study demonstrated different spectra of viral strains of HCV E1-E2 isolates showing synonyms and non-synonyms substitutions, finding glycosylation sites and different epitope domains that could be used to produce monoclonal or polyclonal antibodies targeting both linear and conformational epitopes of envelope glycoprotein E2 that have been shown to inhibit cellular binding of HCV-LP (HCV- Leucoplasts), entry of HCVpp (HCV pseudo-particles) and infection of HCVcc (HCV cell culture).

In conclusion, this study is considered as an important research tool for the development of vaccine trials in Egypt and would help in explaining the existing intra-individual variations of patients in response to the existing drugs in the local market.

\section{Acknowledgements}

To the techniques in Microbial Biotechnology Department

\section{Authors' Contributions}

Omran M. performed the majority of the experiments and wrote the manuscript. Nabil W. performed the majoriy of molecular experiments. Youssef S. coordinated in some of the molecular experiments. El-Sayed M. shared in revising the paper. El-Awady M. involved in writing and revising the manuscript.

\section{Financial Disclosure}

The work is funded through the National Research
Centre.

\section{Funding/Support}

This study was supported financially by Academy of Scientific Research through National Research Centre.

\section{References}

1. Hoofnagle JH. Course and outcome of hepatitis C. Hepatology. 2002;36(5 Suppl 1):S21-9.

2. Abdel-Hamid M, El-Daly M, Molnegren V, El-Kafrawy S, Abdel-Latif $S$, Esmat G, et al. Genetic diversity in hepatitis C virus in Egypt and possible association with hepatocellular carcinoma. J Gen Virol. 2007;88(Pt 5):1526-31.

3. Simmonds P, Liang TJ, Jay HH. 3 Hepatitis C virus genotypes. In: Simmonds P, Liang TJ, Jay HH, editors.Biomedical Research Reports.Academic Press; 2000. p. 53-70.

4. Kato N. Molecular virology of hepatitis C virus. Acta Med Okayama. 2001;55(3):133-59.

5. Zein NN. Clinical significance of hepatitis $\mathrm{C}$ virus genotypes. Clin Microbiol Rev. 2000;13(2):223-35.

6. Mondelli MU, Cerino A, Segagni L, Meola A, Cividini A, Silini E, et al. Hypervariable region 1 of hepatitis $C$ virus: immunological decoy or biologically relevant domain? Antiviral Research 2001;52(2):153-9.

7. El-Awady M, Youssef S, Omran M, Tabll A, El Garf W, Salem A. Soluble egg antigen of Schistosoma Haematobium induces HCV replication in PBMC from patients with chronic HCV infection. BMC Infect Dis. 2006;6(1):1-7.

8. Ohno O, Mizokami M, Wu RR, Saleh MG, Ohba K, Orito E, et al. New hepatitis $\mathrm{C}$ virus (HCV) genotyping system that allows for identification of HCV genotypes 1a, 1b, 2a, 2b, 3a, 3b, 4, 5a, and 6a. J Clin Microbiol.1997;35(1):201-7.

9. Notices. Cladistics. 1989;5(2):163-6

10. Yusim K, Kesmir C, Gaschen B, Addo MM, Altfeld M, Brunak S, et al. Clustering patterns of cytotoxic T-lymphocyte epitopes in human immunodeficiency virus type 1 (HIV-1) proteins reveal imprints of immune evasion on HIV-1 global variation. J Virol. 2002;76(17):8757-68.

11. Farci P, Purcell RH. Clinical significance of hepatitis C virus genotypes and quasispecies. Semin Liver Dis. 2000;20(1):103-26.

12. Timm J, Roggendorf M. Sequence diversity of hepatitis $C$ virus: implications for immune control and therapy. World J Gastroenterol. 2007;13(36):4808-17

13. Quer J, Murillo P, Martell M, Gomez J, Esteban JI, Esteban R, et al. Subtype mutations in the envelope 2 region including phosphorylation homology domain of hepatitis $C$ virus do not predict effectiveness of antiviral therapy. J Viral Hepat. 2004;11(1):4554.

14. Le Guillou-Guillemette H, Vallet S, Gaudy-Graffin C, Payan C, Pivert A, Goudeau A, et al. Genetic diversity of the hepatitis C virus: impact and issues in the antiviral therapy. World J Gastroenterol. 2007;13(17):2416-26.

15. Cannon NA, Donlin MJ, Fan X, Aurora R, Tavis JE. Hepatitis C virus diversity and evolution in the full open-reading frame during antiviral therapy. PLoS One. 2008;3(5).

16. Omran MH, Youssef SS, El-Garf WT, Tabll AA, Bader-Eldin NG, Ate $\mathrm{K}$, et al. Phylogenetic and Genotyping of Hepatitis $\mathrm{C}$ Virus in Egypt. Australia J Basic Appl Sci. 2009;3(1):1-8.

17. Malta Fde M, Medeiros-Filho JE, Azevedo RS, Goncalves L, Silva LC, Carrilho FJ, et al. Sequencing of E2 and NS5A regions of HCV genotype 3a in Brazilian patients with chronic hepatitis. Mem Inst Oswaldo Cruz. 2010;105(1):92-8.

18. von Messling V, Cattaneo R. N-linked glycans with similar location in the fusion protein head modulate paramyxovirus fusion. JVirol. 2003;77(19):10202-12.

19. Al-Qahtani AA, Rubino S, Al-Ahdal MN. Sequence variation of the HVR1 region of Hepatitis $C$ virus in response to interferon-alpha and ribavirin treatment. J Infect Dev Ctries. 2011;5(5):370-6.

20. Goffard A, Dubuisson J. Glycosylation of hepatitis $C$ virus enve- 
lope proteins. Biochimie. 2003;85(3-4):295-301.

21. Helle F, Goffard A, Morel V, Duverlie G, McKeating J, Keck ZY, et al. The neutralizing activity of anti-hepatitis $\mathrm{C}$ virus antibodies is modulated by specific glycans on the E2 envelope protein.J Virol. 2007;81(15):8101-11.

22. Goffard A, Callens N, Bartosch B, Wychowski C, Cosset FL, Montpellier $\mathrm{C}$, et al. Role of $\mathrm{N}$-linked glycans in the functions of hepatitis C virus envelope glycoproteins. J Virol. 2005;79(13):8400-9.

23. Hangartner L, Zinkernagel RM, Hengartner H. Antiviral antibody responses: the two extremes of a wide spectrum. Nat Rev Immunol. 2006;6(3):231-43.

24. Zhong J, Gastaminza P, Cheng G, Kapadia S, Kato T, Burton DR, et al. Robust hepatitis $C$ virus infection in vitro. Proc Natl Acad Sci $U$ S A. 2005;102(26):9294-9.

25. Genovese D, Dettori S, Argentini C, Villano U, Chionne P, Angelico $\mathrm{M}$, et al. Molecular epidemiology of hepatitis $\mathrm{C}$ virus genotype 4 isolates in Egypt and analysis of the variability of envelope proteins E1 and E2 in patients with chronic hepatitis. J Clin Microbiol. 2005;43(4):1902-9.
26. Owsianka A, Tarr AW, Juttla VS, Lavillette D, Bartosch B, Cosset FL, et al. Monoclonal antibody AP33 defines a broadly neutralizing epitope on the hepatitis C virus E2 envelope glycoprotein. J Virol. 2005;79(17):11095-104.

27. Zhang P, Wu CG, Mihalik K, Virata-Theimer ML, Yu MY, Alter HJ, et al. Hepatitis $C$ virus epitope-specific neutralizing antibodies in Igs prepared from human plasma. Proc Natl Acad Sci U S A. 2007;104(20):8449-54.

28. Lavillette D, Pecheur EI, Donot P, Fresquet J, Molle J, Corbau R, et al. Characterization of fusion determinants points to the involvement of three discrete regions of both E1 and E2 glycoproteins in the membrane fusion process of hepatitis $\mathrm{C}$ virus. J Virol. 2007;81(16):8752-65.

29. Cormier EG, Tsamis F, Kajumo F, Durso RJ, Gardner JP, Dragic T. CD81 is an entry coreceptor for hepatitis C virus. Proc Natl Acad Sci U S A. 2004;101(19):7270-4.

30. Thomson M, Liang TJ, Liang TJ, Jay HH. I Molecular biology of hepatitis $\mathrm{C}$ virus. In: Thomson $\mathrm{M}$, Liang TJ, Liang TJ, Jay $\mathrm{HH}$ editors.Biomedical Research Reports.Academic Press; 2000. p.1-I. 